\title{
Challenges Faced by Mexican Americans when Accessing Mental Health Care Service Utilization along the South Texas - Mexico border
}

\author{
Romeo Escobar*, PhD, LCSW, ACSW, John M. Gonzalez, PhD, LMSW, Denise A. Longoria, PhD, LCSW-S, Nelda \\ Rodriguez, LMSW
}

School of Social Work, University of Texas Rio Grande Valley, 1201 W. University Dr., Edinburg, Texas 78539, United States.

Article Details
Article Type: Research Article
Received date: $21^{\text {st }}$ December, 2020
Accepted date: $21^{\text {th }}$ January, 2021
Published date: $22^{\text {nd }}$ January, 2021
"Corresponding Author: Romeo Escobar, PhD, LCSW, ACSW, Assistant Professor, School of Social Work, University of
Texas Rio Grande Valley, 1201 W. University Dr., Edinburg, Texas 78539, United States. E-mail: romeo.escobar@utrgv.edu
Citation: Escobar, R., Gonzalez, J.M., Longoria, D.A., \& Rodriguez N. (2021). Challenges Faced by Mexican Americans
when accessing Mental Health care service utilization along the South Texas - Mexico border. J Ment Health Soc Behav
$3(1): 128$. https://doi.org/10.33790/jmhsb1100128
Copyright: C2021, This is an open-access article distributed under the terms of the Creative Commons Attribution License
4.0, which permits unrestricted use, distribution, and reproduction in any medium, provided the original author and source are
credited.

\begin{abstract}
The purpose of this study was to explore and describe the challenges Mexican Americans face when accessing mental health care service utilization along the South Texas-Mexico border. Disparities in access and use of health and mental health services have led Latinos to be disproportionately represented among those most at-risk for chronic health and mental health illnesses and less likely to receive guideline congruent care. Research on mental health service utilization has documented the presence of economic, cultural, and structural barriers that contribute to the underutilization of health services by Latinos. Researchers conducted three focus groups with 25 mental health providers in the South Texas-Mexico border region. Researchers followed a semi-structured question list and probed for detail from group participants. Kleinman's Explanatory Model [1] guided the question list. The researchers used ethnographic content analysis to analyze the transcripts. Providers reported several challenges when it came to accessing mental health services. The challenges included limited access to insurance, minimal knowledge of mental health, lack of family involvement, few providers, medication management, stigma, and culture.
\end{abstract}

\section{Introduction}

Accessing mental health care should be available to anyone in need of these services; however, for some individuals accessing these services can be a great challenge. The purpose of this study was to explore and describe the challenges Mexican Americans face when accessing mental health care service utilization along the South Texas-Mexico border. For purposes of this article, the term Mexican American has been used in relation to data obtained by the authors; however, the data obtained from the literature review may be more inclusive of other Latinos. Therefore, both terms may be used interchangeably. Disparities in access and use of health and mental health services have led Latinos to be disproportionately represented among those most at-risk for chronic health and mental health illnesses and less likely to receive guideline congruent care. Latinos are less likely to receive mental health treatment when compared to other ethnic groups $[2,3]$. Research on mental health service utilization has documented the presence of economic, cultural, and structural barriers that contribute to the underutilization of health services by Latinos when compared to other ethnic groups [4].

\section{Literature Review}

Latinos diagnosed with mental illness tend to not access or use mental health services available to them [5-8]. Villatoro, et al. [9] further added that Latinos are the largest and fastest growing racial/ ethnic minority group in the United States, and they traditionally do not access these services. The underutilization of mental health services among Latinos is in part a function of structural factors that affect their opportunities. In light of this projected population growth and the activation of the Affordable Care Act in 2010, there is an emerging necessity to be better prepared to address the mental health needs of Latinos, particularly in public mental health services [9]. Similarly, Weissman et al. [10] studied health care access and utilization among adults with serious psychological distress by race, ethnicity, and gender and found that Latinos along with nonHispanic blacks faced increased barriers to care and lower utilization compared to non-Hispanic whites. VanderWielen et al, [11] studied racial and ethnic disparities in access to nearby behavioral health care and primary care and found that Hispanics were less likely to have geographically proximate behavioral health professionals; therefore, underutilization was prominent.

In line with the documented barriers and disparities to mental health services, a lower number of Mexican Americans participate in the health care arena and are not compliant with their medical regimen. More important is the lack of mental health research of Mexican Americans along the southern region, especially since it is difficult to determine the true extent of mental health needs because there is a lack of data and information. Research highlights the need for understanding relevant factors that affect positive outcomes and led to pervasive disparities in mental health service use among this population. Extensive research on mental health service utilization has consistently documented the presence of economic, cultural, and structural barriers that contribute to the underutilization of health services by Latinos compared to the general population and other ethnic groups $[4,5,9,12,13]$.

The U.S. Census Bureau [14] estimated that Latinos will comprise almost one-third of the U.S. population by 2060. In Texas, the projections have Latinos becoming the majority between 2026 and 2027 with the increase expected to be larger along the Texas-Mexico border $[14,15]$. Along the border, the poverty rate is $30-35 \%$ of the population. This is significant because according to Hudson [16], people who were impoverished were three times more likely to suffer from a mental illness.

Considering the central role of familismo in Latin culture, it is important to assess the extent to which familismo affects mental health help-seeking [17]. No other element of the Latino culture is as significant as the family and its extended network of immediate 
and fictive kin; thus, (the family) includes individuals who are blood related and some in the community that are not blood related. La familia is comprised of a nuclear family and an extended network of immediate and fictive members, bound by strong feelings of loyalty, identification, attachment, mutual support, and solidarity among its family members [17-19].

Latinos are often dependent on family support and friends to meet their healthcare needs $[17,20]$. Keeping physical and emotional problems in the family is a norm that contributes to their reluctance to take advantage of available mental healthcare services [21]. With respect to mental illness, the issue of mistrust is an added factor. In one study, Schwarzbaum [22] investigated potential barriers to counseling specifically for Hispanics who were identified as having a low income. Schwarzbaum found Latinos from low-income families were more likely to have a sense of mistrust with nonHispanic counselors primarily based on fear of being labeled (crazy) or running the risk of being deported. In short, family caregiving can be a double-edged sword in times of need for health services. In a similar study by Hodgkinson et al. [23] found that poverty is a common experience for many children and families in the United States. Living in a poor or low-income household has been linked to poor health and increased risk for mental health problems in both children and adults that can persist across the life span (Hodgkinson et. al., [23]. The family can be a great facilitator in helping older Latino family members access needed resources including mental health services, yet at other times, the family can be a barrier to accessing these services.

The purpose of this study was to explore and describe the challenges Mexican Americans face when accessing mental health care service utilization along the South Texas-Mexico border. Understanding these challenges will help mental health care professionals work with Mexican Americans in helping families and caregivers to cope with their ill relatives.

\section{Methods}

The ways in which Mexican Americans experience mental illness and find meaning for their mental health experiences are best studied using a qualitative approach. Therefore, consistent with Padgett's [24] recommendations, a focus group qualitative methodological approach was used to study challenges Mexican Americans face when accessing mental health care service utilization along the South Texas-Mexico border. As a first step in studying this hard-to-reach population, researchers decided to seek information from treatment professionals working with Mexican American clients. The study relied on focus groups to gather practitioners' perceptions of clients' and family mental health treatment experiences.

The researchers recruited 25 mental health practitioners (social workers, licensed professional counselors and psychologists $(\mathrm{N}=25)$ from the South Texas-Mexico border to attend one of three focus groups to solicit their perceptions regarding challenges faced by Mexican American clients and families in accessing mental health services. The purpose of the focus groups in which they participated was to generate diverse ideas. For this reason, all members were encouraged to participate. Researchers collected narratives in summer 2016. The researchers held the focus group discussions at three distinct locations alongthe South Texas-Mexico border: McAllen/Edinburg, Brownsville, and Laredo.

The South Texas-Mexico border is predominantly MexicanAmerican, and many residents can speak English and Spanish; moreover, some residents speak both languages interchangeably at the same time. In general, focus groups should occur in nonthreatening environments with a group of individuals who share certain characteristics to allow for a good group dynamic and greater self-disclosure $[25,26]$.

After receiving IRB approval, researchers conducted focus groups in private conference rooms at three agency facilities. The researchers obtained informed consent from all participants in the study. Focus group participants sat around the conference room table and were able to see one another. Two moderators conducted focus groups in the language that the participants preferred (English, Spanish, or a combination of both). Researchers had experience facilitating focus groups. Focus group discussions lasted an average of 60 minutes, and they were recorded for transcription purposes $[25,26]$.

The researchers followed a list of semi- structured guiding questions and probed for examples, clarification, and/or details from group participants. The focus group questions centered on the challenges MexicanAmerican families experienced in accessing mental health services. The questions were adapted from Kleinman's explanatory model [1], which is often used to examine help-seeking behaviors and culture.

According to Kleinman, an explanatory model encompasses the notions a person has about an episode of illness and its treatment as delivered by all those who engage in the clinical process. Explanatory models examine people's cognitive processes based on their cultural knowledge and idiosyncratic experiences. Popular culture and the media inform explanatory models as do the health care culture and the social network of the individual. These elements guide the interpretation and action concerning health. Individuals form explanatory models to cope with a specific health problem. Explanatory models determine what important clinical evidence is and how it is organized and interpreted for treatment approaches. Explanatory models help patients and families in making sense of illness episodes [1].

Analysis began immediately after the focus group discussions. Researchers did not need to make any adjustments to the guiding questions or research protocol. Researchers performed all the content analysis, including conceptual improvement and identification of pertinent categories. Finally, the researchers integrated and interpreted the study findings. The researchers used ethnographic content analysis [27-29] to analyze the transcripts of the practitioners' perceptions of the challenges Mexican Americans face when accessing mental health care service utilization along the South Texas-Mexico border. Ethnographic content analysis centered on the identification of concepts, collection of narratives, and emergent patterns and categories through repeated study of the content or text. Ethnographic content analysis followed a recursive and reflexive movement between the narratives and the interpretation of those narratives by the different members of the research team. The aim was to be systematic and analytic but not rigid. The process was oriented toward clear description and definition. According to Altheide [29], the process of qualitative document analysis includes examining the content of the narratives to allow emergence, improvement, or collapsing of added categories.

Ethnographic content analysis enabled researchers to organize the data. Four coders independently coded each transcript one by one. The codes identified keywords, phrases, meanings, and concepts. The authors then met and discussed similarities and potential connections among keywords, phrases, and concepts, within and across the focus groups. Coding discrepancies were reconciled through these discussions. All codes and their meanings are presented in the Findings section per category. This is a descriptive analysis and was not concerned with prevalence of mental distress.

To enhance the trust worthiness of the collected narratives, the researchers also conducted individual interviews with three of the mental health professionals who also took part in the focus groups to provide an opportunity for "member checking." The interviews lasted 30 minutes. Member checking, or returning to the field to verify the analysis, ensures that the interpretation is on the right track [30]. In the analytic process, researchers also engaged in modified member checking with some focus group participants by asking them 
to explain further any material that was unclear regarding the meanings and the themes that emerged from the transcriptions/ interview recordings.

\section{Findings}

In this study, the researchers explored the challenges Mexican Americans from the South Texas-Mexico border face when accessing mental health services. Culture was a predominant theme reported by the providers. Culture and poverty had an influence in the health behaviors of Mexican Americans when faced with challenges to access treatment. These challenges included limited access to insurance, minimal knowledge of mental health and resources, stigma, few providers, culture, medication management, lack of family involvement, and transportation.

\section{Insurance}

One of the barriers Mexican Americans encounter trying to access health care is being uninsured. While many clinics have tried helping the uninsured by linking them to indigent program(s), there is still the issue of whether they qualify or not.

If they are uninsured, we will link them to the indigent program and try to utilize those resources.

Some clinics such as 'SuClinica' in the Rio Grande Valley have sliding scales that determine the amount they will have to pay based on their income, which makes health care a bit more accessible to some.

...becoming a patient of SuClinica, if they don thave any insurance, they will go ahead and be registered on a sliding scale.

However, a challenge faced with this system is a long waiting period if the clinic is at its capacity limit.

The clinics right now are to the limit already. There is a waiting period.

The middle class are the ones that have a gap in resources. They might have Medicare, but no supplemental insurance and their income level disqualifies them from Medicaid.

If you have low income, you might have Medicaid and other. So, it's really the middle class that is underserved.

\section{Lack of mental health knowledge}

It is not uncommon for people to have minimal knowledge about mental health and resources. People know to go to a Primary Care Physician when they feel sick. However, when it comes to not feeling well emotionally, people do not have the knowledge of the mental health and resources available.

If physically, we don't feel well, we know to go see a PCP for example. But if emotionally, we don't feel like we are stable, no one thinks about going to see a mental health professional.

\section{Stigma}

Providers reported mental health stigma is an immense challenge for clients and their families, specifically considering the strong relationship of "being loco (crazy)" has within the Mexican American culture. No one wants to be thought of as "loco/crazy" nor do they want the term associated with their family.

The word loco always comes up, 'no estoy loco. 'So, the older, the younger ones they'rekinda a little bit more open, but the older ones are a little bit like, they even tell me well just the fact that I'm here sitting with you is confirming that I'm crazy.

Providers reported the family can be instrumental in helping their loved one overcome stigma through involvement and support to not only seek mental health services but also to receive treatment.

I've seen the family be instrumental they step in and say, "no ma, pero pos this is the medication that is supposed to help you with that, you know we are only trying to help you"... and, and so they encourage their loved one.

\section{Few mental health providers}

Participants in the study reported there are few mental health providers in the South Texas-Mexico border which makes it a big challenge for Mexican Americans to access mental health services. Some agencies will contract with psychiatrists, and the psychiatrist will be available for a brief time to see patients resulting in a long wait for a diagnosis and treatment.

There'sa limited number of psychiatrists out in the community and we did, we were able to contract one as a consulting psychiatrist. He can only come in, he only comes in like one hour and will see two patients, so ugh the waiting period to see a psychiatrist."

\section{Lack of Medication}

Here at the South Texas-Mexico border, one challenge that is faced is the access to the correct medication. Participants reported some patients who seek mental health care often cross the border for medications which comes with its own risks and barriers. These patients often end up buying and/or taking the wrong medication for their diagnosis.

Many times, the medication doesn't match the actual, ugh, diagnosis so now you're talking about, you know, improper treatment and ineffective treatment.

Participants reported families will often obtain medications without a prescription. It is almost like "a family pharmacy" and they manage their medications themselves. At times, patients will use medications the family has. Medications come "from mom, dad, and other siblings." And (they do this) again, to try to "self-medicate" and some patients tend to "go into Mexico and get medication there." So that is a very big problem.

Another challenge reported by participants was medication not being readily available for those who are uninsured such as at an established mental health facility. This was a challenge for families and providers.

The pharmacy doesn't have all the available medications like Tropical Texas would.It's a little bit of a barrier for people that don't have insurance.

\section{Family involvement}

The patient responds well when the family is involved in the treatment; however, getting the family involved is often a challenge. Family members might be reluctant to participate or take the responsibility for a loved one.

The family members have very limited involvement; I mean you're trying to get them in as much as we can, but they expect the professional to do most of it most of the leg work most of the umm any kind of, any kind of work.

A lack of family support was reported for some of the patients, even though there was support from the family at home; however, it did not always transcend to the care and maintenance of the patient's mental health care.

I mean these patients that I've had decline, they usually come alone. There, there isn't really family there with them.

This can be attributed to families growing tired of helping their family member. The family members tryto help their loved one and still no change.

The family is already tired of them.... which becomes another challenge.

Family members may reduce their involvement particularly when the family's help makes a slight difference in their loved one's life. As they age, it becomes even more difficult to care for their loved one.

Depending on the age of the individual too that has a lot to play with it, if umm, you know, the person has been homeless for a while 
or broke a lot of their bridges with their families sometimes they don'twanna be involved.

Family members begin to stray away from helping their family member. This impacts the level of family involvement with the agency and treatment team. This creates a challenge for the treatment team.

\section{Limited Transportation}

Limited transportation can be a challenge for patients or clients. Some patients might not have transportation or rely on a family member to help with transportation. Whether it be inability or unwillingness, it poses an added barrier to the patient.

They don't want to take the transportation, or their family members are too busy working or going to school and who's going to take them. It doesn't sound that important, but it is significant and it's a big thing.

It is also a challenge for families who want to be involved with the care of their loved but cannot make appointments because they lack transportation or finding a family member who is available to transport their loved one.

A lot of the contacts we have with families are contacts over the phone. You know, a lot of the families don't have transportation to get in so what we do is talk to them on the phone to get specific history from them there and then talk to them about where they were living, where they want to go, what resources does the family has.

\section{Discussion}

For many Mexican Americans, there is a pervasive lack of understanding of mental illness and its consequences. Thus, this lack of understanding becomes a deterrent factor and a big challenge for Mexican Americans in seeking mental health services. For Mexican Americans to increase their involvement in seeking mental health treatment, they must first have sufficient knowledge about mental illness and its consequences. An additional challenge for Mexican Americans identified in this study is the lack of insurance to seek mental health treatment. Often, this results in the individual not getting the necessary mental health care needed.

Invariably when an individual is not in treatment, their behavior may not be considered appropriate by others which results in the individual being labeled as "crazy". Often, it isn't that the individual is "crazy" per se, but the lack of understanding of the behavior being manifested by the individual immediately is associated with the stigma of "being crazy". In the Mexican American culture, there is a strong relationship with this stigma which deters individuals requiring mental health care from seeking treatment. According to the National Institute of Mental Health [31], 1 in 5 individuals in any given population is afflicted with mental illness which translates to $20 \%$. The Rio Grande Valley has a population of 1.3 million with the majority being Mexican Americans. Therefore, $20 \%$ of this population $(260,000)$ may be afflicted with mental illness.

A big challenge with respect to the population in the Rio Grande Valley is the lack of sufficient mental health providers to meet the mental health care needs of individuals suffering from mental illness. Often, with few mental health providers being available to provide this necessary treatment, additional challenges surface such as lack of transportation and lack of family involvement. Transportation becomes an issue for many Mexican Americans when they must travel approximately anywhere between 30 to 40 miles to obtain the mental health services. In addition, the lack of family involvement becomes an insurmountable issue for the individual with mental illness. Finally, for many Mexican Americans, the need to adhere to appropriate psychotropic medications is a deterrent factor in the emotional well-being of the individual.

\section{Limitations}

A limitation of this study is generalizability due to the qualitative nature of the data collection. The study sample was purposive, which makes it difficult to generalize it to all Mexican Americans. However, findings do provide insights into the experiences of Mexican Americans and the associated cultural norms of those communities associated with the practitioners who participated. Another limitation of this study is the findings were based on the practitioners' perceptions rather than challenges identified by the clients themselves.

\section{Implications and recommendations}

This study gives the South Texas-Mexico border mental health care community insights into the contributors of mental health care utilization for Mexican Americans. These insights will also help the South Texas-Mexico border mental health care community to improve services and service delivery.

\section{Education of mental illness and treatment services}

Recommendations for social work and health care providers are to increase education on mental illness and services to Mexican Americans and their families. Providing education will help increase the understanding of mental illness and mental health services. For example, Mexican Americans and their families need education concerning the insurance benefits for which they qualify for mental health services, plus general information about mental health.

Another need is to reduce stigma of mental illness and mental health treatment. To do this, there is a need to educate the community on mental illness and mental health services to help eliminate confusion of what mental illness is. Today, understanding mental illness is a part of life. Targeting young adults will help reduce the stigma of mental illness and provide the opportunity to educate the community about mental illness and mental health services.

\section{Recruitment of bilingual/bicultural social workers}

Finally, to understand the complexities of the Mexican American culture and the value system of the Mexican American population especially when dealing with mental health services, there is a need to recruit bilingual/bicultural social workers in mental health facilities. Bilingual/bicultural social workers will minimize the misunderstanding of the Mexican American culture with respect to mental health utilization and the related issues surrounding this delicate topic. Bilingual/bicultural social workers who can relate to the Mexican American population and its cultural belief system will also help minimize the stigma associated with mental health treatment and hopefully increase utilization of this much needed service along the South Texas-Mexico border.

\section{Conclusion}

Future research should aim to include Mexican Americans and respective families. Additionally, studies should focus on several questions which come to mind regarding studying Mexican Americans and their families. What factors affect accessing mental health care services? How does the Mexican American cultural belief system impact the decision to seek treatment services? How do Mexican Americans and their families cope with stigma related to mental health? Is there a set of coping skills that Mexican Americans and their families use to access mental health services?

The challenges faced by Mexican Americans in seeking mental health treatment can be overwhelming and taxing. Confronting these challenges merits a lot of commitment and dedication from both the mental health care community and the Mexican American population working together. However, appropriate resources, sensitivity, and understanding of the culture of the Mexican American population will help in overcoming these challenges, increase the utilization of mental health services, and diminish the existing disparity in mental health treatment access. The above recommendations made may not only be applicable to the South Texas-Mexico border Mexican American community, but other Mexican American communities in the U.S. who may be facing the same or similar challenges.

Conflict of interests: The authors declare no conflict of interest. 


\section{References}

1. Kleinman, A. (1980). Patients and healers in the context of culture: An exploration of the borderland between anthropology, medicine, and psychiatry. Berkeley \& Los Angeles: University of California Press.

2. Alegria, M., Canino, G., Rios, R., Vera, M., Calderon, J., Rusch, D., \& Ortega, A. (2002). Inequalities in use of speciality mental health services among Latinos, African Americans, and nonLatino Whites. Psychiatric Services, 53 (12) 1547-1555.

3. Miranda, J. \& Cooper, L. A. (2004). Disparities in care for depression among primary care patients. Journal of General Internal Medicine 19, 120-126.

4. Marin, H., Escobar,J. I. \& Vega, W. A. (2006). Mental Illness in Hispanics: A review of the literature. Focus, 4 (1): 23-37.

5. Barerra. I., \& Longoria, D. (2018). Examining cultural mental health barriers among Latinos. Journal of Leadership, Equity, and Research. Vol. 4(1).

6. Alegría, M.A., Chatterji, P., Wells,K., Cao, Z., Chen, C.N., Takeuchi, D...Meng, X. L. (2008). Disparity in depression treatment among racial and ethnic minority populations in the United States. Psychiatric Services, 59, 1264 - 1272.

7. Blanco, C., Patel, S.R., Liu, L., Jiang, H., Lewis-Fernandez, R., Schmidt, A. B...Olfson, M. (2007). National trend in ethnic disparities in mental health care. Medical Care, 45, 1012 - 1019.

8. Cook, B., McGuire, T., \& Miranda, J. (2007). Measuring trends in mental health care disparities, 2000-2004. Psychiatric Services, 58, 1533-1540.

9. Villatoro, A. P., Dixon, E., \& Mays, V. M. (2016). Faith-Based organizations and the Affordable Care Act: Reducing Latino mental health care disparities.Psychological Services, Vol. 13(1), 92-104.

10. Weissman, J., Russel, D., Jay, M., \& Malaspina, D., (2018). Racial, ethnic, and gende disparities in healthcare access and use among U. S. adults with psychological distress. Psychiatric Services, 69(5), 517-522.

11. VanderWeilen, L, Gilchrist, E., Nowels, M., Petterson, S., Rust, G., \& Miller, B., (2015). Not enough: Racial and ethnic disparities in access to nearby behavioral health care and primary care. Journal of Health Care for the Poor \& Underserved. 26(3), 1032-1048.

12. Rodriguez-Keys, E., \&Piepenbring, J. (2017). Building a bicultural bridge. Smith CollegeStudies in Social Work, 87(2-3), 153-169.

13. Atdjian, S., \& Vega, W. A. (2005). Disparities in mental health treatment in U. S. racial and ethnic minority groups: Implications for psychiatrists. Psychiatric Services, 56 (12), 1600-1602.

14. U.S. Census Bureau. (2016.) U.S. Census Bureau projections show a slower growing, older more diverse nation a half century from now. Retrieved from http://www.census.gov/newsroom/ releases/archives/population/cb12-243.html

15. Texas State Data Center and Office of the State Demographer (2006). A summary of the Texas challenge in the twenty-first century: Implications of population change for the future of Texas, 2006. Census of Population, U.S. Bureau of the Census as compiled by the Texas State Data Center, The University of Texas at San Antonio. Retrieved from http://txsdc.utsa.edu/ pubsrep/pubs/txchal.php

16. Hudson, C. G. (2005). Socioeconomic status and mental illness: Tests of the social causation and selection hypotheses [Electronic version]. American Journal of Orthopsychiatry, 75, 3-18.
17. Villatoro, A. P., Morales, E. S., \&Mays, V. M. (2014). Family culture in mental health help-seeking and utilization in a nationally representative sample of Latinos in the United States: The NLAAS. American Journal of Orthopsychiatry, 84(4), 353375.

18. Gonzalez, N. A., Germán, M., \&Fabrett, F. C. (2012) US Latino Youth. In Chang, E. C. \& Downey, C. A. (Eds) Handbook of Race and Development in Mental Health. Springer. Pp. 307327.

19. Organista, K. (2007). Solving Latino psychosocial and health problems: Theory, practice and populations. Hoboken, NJ; John Wiley and Sons, Inc.

20. Angel, J. L., Angel, R. J., McClellan, J. L., \& Markides, K. S. (1996). Nativity, declining health and preferences in living arrangements among elderly Mexican Americans: Implications for long-term care. Gerontologist, 36(4), 464-473.

21. Magilvy, J. K., Congdon, J. G., Martinez, R. J., Davis, R., \& Averill, J. (2000). Caring for our own; health care experiences of rural Hispanic elders. Journal of Aging Studies, 14(2), 171-191.

22. Schwarzbaum, S. E. (2004). Low-income Latinos and dropout: Strategies to prevent dropout. Journal of multicultural counseling and development, 32, 296 - 306. Retrieved January 3, 2008, from Academic Search Premier database.

23. Hodgkinson, S., Godoy, L., Beers, L. S., \& Lewin, A. (2017). Improving mental health access for low-income children and families in the primary healthcare setting. Pediatrics, 139(1).

24. Padgett, D. (2016). Qualitative methods in social work research. Sage Publications: New York, NY.

25. Krueger, R. A. \& Casey, M. A. (2015). Focus groups. A practical guide for applied research (5th ed.). Thousand Oaks: Sage.

26. Krueger, R. A. \& Casey, M. A. (2000). Focus groups. A practical guide for applied research (3rd ed.). Thousand Oaks: Sage.

27. Altheide, D. L. \& C. J. Schneider (2013). Qualitative Media Analysis: Qualitative Research Methods 2nd ed. Sage Publications: Thousand Oaks.

28. Stark, R. \& Roberts, L. (2005). Content analysis. Chapter 19 pp. 303-313. In Social Work Research and Evaluation: Quantitative and qualitative approaches. 7th ed. Grinnell, R. m. \&Unrau. Y. A. Eds. Oxford Press: New York.

29. Altheide, D. L. (1996). Qualitative Media Analysis: Qualitative Research Methods Serious 38. Sage Publications: Thousand Oaks.

30. Lincoln, Y. S., \& Guba, E. G. (1985). Naturalistic inquiry. Beverly Hills, CA: Sage Publications, Inc. Newbury Park, CA: Sage.

31. National Institute of Mental Health (2019). Mental Illness. Retrieved from https://www.nimh.gov/heatlh/statistics/mentalillness.shtml. 